\title{
CALENDAR OF MEETINGS
}

The Call for Papers and Calendar sections list conferences, symposia, and workshops that are of potential interest to AMS members. Complete information about events listed in the calendar can be found on the meetings page of the AMS website, www.ametsoc.org. New additions to the calendar are highlighted.

To list an event in the calendar, please submit the event name, dates, location, and deadlines for abstracts, manuscripts, and preregistration to amsmtgs@ametsoc.org. For a submission to appear in a given issue, it must be submitted at least eight weeks prior to the month of publication (that is, to appear in the March Bulletin, the submission must be received by I January).

\section{AMS MEETINGS}

\section{8}

\section{OCTOBER}

29th Conference on Severe Local Storms, 22-26 October, Stowe, Vermont Abstract deadline: 15 June 2018

Preregistration deadline: 31 August 2018 Extended abstract deadline: 23 Nov. 2018 Initial announcement published: Oct. 2017

\section{Automated Vehicles and Meteorology Summit, 23-24 October, Washington, D.C.}

Preregistration deadline: 24 September 2018 Initial announcement published: May 2018

\section{9}

\section{JANUARY}

AMS Short Course: Integrating NWP System Components Using Container Technology, 5 January, Phoenix, Arizona

Preregistration deadline: 3 December 2018 Initial announcement published: Oct. 2018

AMS Short Course: Tutorial on How to Build a 3D-Printed Automatic Weather Station, 5 January, Phoenix, Arizona Preregistration deadline: 3 December 2018 Initial announcement published: Oct. 2018

AMS Short Course: Python for Synoptic Meteorology Using Metpy, 6 January, Phoenix, Arizona

Preregistration deadline: 3 December 2018 Initial announcement published: Oct. 2018
AMS Short Course: An Introduction to Using the NASA Giovanni System for Multidisciplinary Research and Applications, 6 January, Phoenix, Arizona Preregistration deadline: 3 December 2018 Initial announcement published: Oct. 2018

AMS Short Course: Applying JPSS Data Products to Better Forecast Challenging Weather Events, 6 January, Phoenix, Arizona

Preregistration deadline: 3 December 2018 Initial announcement published: Oct. 2018

AMS Short Course: GOES-R Series: Forecasting Applications, 6 January, Phoenix, Arizona

Preregistration deadline: 3 December 2018 Initial announcement published: Oct. 2018

AMS Short Course: Machine Learning in Python for Environmental Science Problems, 6 January, Phoenix, Arizona Preregistration deadline: 3 December 2018 Initial announcement published: Oct. 2018

AMS Short Course: Weather and Phased Radar Polarimetry, 6 January, Phoenix, Arizona

Preregistration deadline: 3 December 2018 Initial announcement published: Oct. 2018

AMS Short Course: A Beginner's Course to Using Python in Climate and Meteorology, 5-6 January, Phoenix, Arizona

Preregistration deadline: 3 December 2018 Initial announcement published: Oct. 2018
AMS Short Course: Using NASA High Performance Computers to Perform Analytics on Large Earth Science Data, 6 January, Phoenix, Arizona Preregistration deadline: 3 December 2018 Initial announcement published: Oct. 2018

AMS Short Course: Introducing the Community Earth System Model, version 2 (CESM2) via an Interactive Hands-On Tutorial Using the Cloud, 6 January, Phoenix, Arizona Preregistration deadline: 3 December 2018 Initial announcement published: Oct. 2018

AMS Workshop on Diversity, Equity and Inclusion for Geoscientists, 6 January, Phoenix, Arizona

Preregistration deadline: 3 December 2018 Initial announcement published: Oct. 2018

19th Presidential Forum, 6 January, Phoenix, Arizona

Preregistration deadline: 3 December 2018

Initial announcement published: Sep. 2018

${ }^{*}$ Richard Anthes Symposium, 6-10 January, Phoenix, Arizona

Abstract deadline: 1 August 2018

Preregistration deadline: 3 December 2018

Extended abstract deadline: 15 Feb. 2019 Initial announcement published: May 2018

${ }^{\star}$ An exhibit program will be held at this meeting. 
${ }^{*}$ Inez Fong Symposium, 6-10 January, Phoenix, Arizona

Session topic proposal deadline: 1 April 2018

Abstract deadline: 1 August 2018

Preregistration deadline: 3 December 2018

Extended abstract deadline: 15 Feb. 2019

Initial announcement published: May 2018

*35th Conference on Environmental Information Processing Technologies, 6-10 January, Phoenix, Arizona

Session topic proposal deadline: 1 April 2018 Abstract deadline: 1 August 2018

Preregistration deadline: 3 December 2018

Extended abstract deadline: 15 Feb. 2019

Initial announcement published: May 2018

*33rd Conference on Hydrology, 6-11 January, Phoenix, Arizona

Session topic proposal deadline: 1 April 2018

Abstract deadline: 1 August 2018

Preregistration deadline: 3 December 2018

Extended abstract deadline: 15 Feb. 2019

Initial announcement published: May 2018

*32nd Conference on Climate Variability and Change, 6-11 January, Phoenix, Arizona

Session topic proposal deadline: 1 April 2018

Abstract deadline: 1 August 2018

Preregistration deadline: 3 December 2018

Extended abstract deadline: 15 Feb. 2019

Initial announcement published: May 2018 *28th Symposium on Education, 6-11 January, Phoenix, Arizona

Session topic proposal deadline: 1 April 2018

Abstract deadline: 1 August 2018

Preregistration deadline: 3 December 2018

Extended abstract deadline: 15 Feb. 2019

Initial announcement published: May 2018

${ }^{\star}$ 24th Conference on Applied Climatology, 6-11 January, Phoenix, Arizona

Session topic proposal deadline: 1 April 2018

Abstract deadline: 1 August 2018

Preregistration deadline: 3 December 2018

Extended abstract deadline: 15 Feb. 2019

Initial announcement published: May 2018

*23rd Conference on Integrated $\mathrm{Ob}$ serving and Assimilation Systems for the Atmosphere, Oceans, and Land Surface (IOAS-AOLS), 6-11 January, Phoenix, Arizona

Session topic proposal deadline: 1 April 2018

Abstract deadline: 1 August 2018

Preregistration deadline: 3 December 2018

Extended abstract deadline: 15 Feb. 2019

Initial announcement published: May 2018

\section{*22nd Conference of Atmospheric Sci-} ence Librarians International, 6-11 January, Phoenix, Arizona

Session topic proposal deadline: 1 April 2018

Abstract deadline: 1 August 2018

Preregistration deadline: 3 December 2018

Extended abstract deadline: 15 Feb. 2019

Initial announcement published: June 2018 *21st Conference on Atmospheric Chemistry, 6-11 January, Phoenix, Arizona

Session topic proposal deadline: 1 April 2018

Abstract deadline: 1 August 2018

Preregistration deadline: 3 December 2018

Extended abstract deadline: 15 Feb. 2019

Initial announcement published: May 2018

*20th Conference on Middle Atmosphere, 6-11 January, Phoenix, Arizona

Session topic proposal deadline: 1 April 2018

Abstract deadline: 1 August 2018

Preregistration deadline: 3 December 2018

Extended abstract deadline: 15 Feb. 2019

Initial announcement published: May 2018

${ }^{\star} 19$ th Conference on Aviation, Range, and Aerospace Meteorology, 6-11 January, Phoenix, Arizona

Session topic proposal deadline: 1 April 2018 Abstract deadline: 1 August 2018

Preregistration deadline: 3 December 2018

Extended abstract deadline: 15 Feb. 2019

Initial announcement published: May 2018

${ }^{*}$ An exhibit program will be held at this
meeting.

\section{STUDENT TRAVEL GRANTS}

Student Travel Grants are available for senior undergraduate and graduate students to attend AMS meetings held in the United States and Canada. The travel grants are available only to members, including student members, of the AMS.

AMS recognizes the considerable benefit that students can gain from attending conferences even if they are not presenting a paper there, and AMS wants to encourage interactions between students and other conference attendees. To this end, travel grants will be awarded to a student who is not presenting a paper at the conference.

Students who are presenting papers and potentially in need of travel support should inquire of the program chair whether any funds will be available for this purpose.

For more information and to complete an application form, please visit the AMS website at www. ametsoc.org. 
${ }^{*} 18$ th Conference on Artificial and Computational Intelligence and its Applications to the Environmental Sciences, 6-11 January, Phoenix, Arizona Session topic proposal deadline: 1 April 2018 Abstract deadline: 1 August 2018

Preregistration deadline: 3 December 2018

Extended abstract deadline: 15 Feb. 2019

Initial announcement published: May 2018

${ }^{\star} 17$ th Symposium on the Coastal Environment, 6-11 January, Phoenix, Arizona

Session topic proposal deadline: 1 April 2018

Abstract deadline: 1 August 2018

Preregistration deadline: 3 December 2018

Extended abstract deadline: 15 Feb. 2019

Initial announcement published: May 2018

${ }^{*}$ 17th History Symposium, 6-11 January, Phoenix, Arizona

Session topic proposal deadline: 1 April 2018

Abstract deadline: 1 August 2018

Preregistration deadline: 3 December 2018

Extended abstract deadline: 15 Feb. 2019

Initial announcement published: May 2018

${ }^{*} 16$ th Conference on Space Weather, 6-11 January, Phoenix, Arizona Session topic proposal deadline: 1 April 2018 Abstract deadline: 1 August 2018

Preregistration deadline: 3 December 2018

Extended abstract deadline: 15 Feb. 2019

Initial announcement published: May 2018

${ }^{\star} 15$ th Annual Symposium on New Generation Operational Environmental Satellite Systems, 6-11 January, Phoenix, Arizona

Session topic proposal deadline: 1 April 2018

Abstract deadline: 1 August 2018

Preregistration deadline: 3 December 2018

Extended abstract deadline: 15 Feb. 2019

Initial announcement published: May 2018
${ }^{\star}$ 15IMPACTS: Major Weather Events and Impacts of 2018, 6-11 January, Phoenix, Arizona

Session topic proposal deadline: 1 April 2018

Abstract deadline: 1 August 2018

Preregistration deadline: 3 December 2018

Extended abstract deadline: 15 Feb. 2019

Initial announcement published: May 2018

${ }^{*}$ 14th Symposium on Societal Applications: Policy, Research and Practice, 6-11 January, Phoenix, Arizona

Session topic proposal deadline: 1 April 2018

Abstract deadline: 1 August 2018

Preregistration deadline: 3 December 2018

Extended abstract deadline: 15 Feb. 2019

Initial announcement published: May 2018

${ }^{*} 11$ th Symposium on Aerosol-CloudClimate Interactions, 6-11 January, Phoenix, Arizona

Session topic proposal deadline: 1 April 2018

Abstract deadline: 1 August 2018

Preregistration deadline: 3 December 2018

Extended abstract deadline: 15 Feb. 2019

Initial announcement published: May 2018

${ }^{\star} 10$ th Conference on Weather, Climate, and the New Energy Economy, 6-11 January, Phoenix, Arizona

Session topic proposal deadline: 1 April 2018

Abstract deadline: 1 August 2018

Preregistration deadline: 3 December 2018

Extended abstract deadline: 15 Feb. 2019

Initial announcement published: May 2018

${ }^{*} 10$ th Conference on Environment and Health, 6-11 January, Phoenix, Arizona

Session topic proposal deadline: 1 April 2018

Abstract deadline: 1 August 2018

Preregistration deadline: 3 December 2018

Extended abstract deadline: 15 Feb. 2019

Initial announcement published: May 2018
${ }^{*}$ Ninth Conference on Transition of Research to Operations, 6-11 January, Phoenix, Arizona

Session topic proposal deadline: 1 April 2018 Abstract deadline: 1 August 2018

Preregistration deadline: 3 December 2018

Extended abstract deadline: 15 Feb. 2019

Initial announcement published: May 2018

${ }^{\star}$ Ninth Conference on the Meteorological Application of Lightning Data, 6-11 January, Phoenix, Arizona

Session topic proposal deadline: 1 April 2018 Abstract deadline: 1 August 2018

Preregistration deadline: 3 December 2018

Extended abstract deadline: 15 Feb. 2019

Initial announcement published: May 2018

*Ninth Symposium. on Lidar Atmospheric Applications, 6-11 January, Phoenix, Arizona

Session topic proposal deadline: 1 April 2018

Abstract deadline: 1 August 2018

Preregistration deadline: 3 December 2018

Extended abstract deadline: 15 Feb. 2019

Initial announcement published: May 2018

${ }^{*}$ Ninth Symposium on Advances in Modeling and Analysis Using Python, 6-11 January, Phoenix, Arizona

Session topic proposal deadline: 1 April 2018

Abstract deadline: 1 August 2018

Preregistration deadline: 3 December 2018

Extended abstract deadline: 15 Feb. 2019

Initial announcement published: June 2018

${ }^{\star}$ Seventh Symposium on the Weather, Water, and Climate Enterprise, 6-11 January, Phoenix, Arizona

Session topic proposal deadline: 1 April 2018 Abstract deadline: 1 August 2018 Preregistration deadline: 3 December 2018 Extended abstract deadline: 15 Feb. 2019 Initial announcement published: May 2018

\footnotetext{
${ }^{\star}$ An exhibit program will be held at this meeting.
} 
* Seventh Symposium on Building a Weather-Ready Nation: Enhancing Our Nation's Readiness, Responsiveness, and Resilience to High Impact Weather Events, 6-11 January, Phoenix, Arizona

Session topic proposal deadline: 1 April 2018 Abstract deadline: 1 August 2018

Preregistration deadline: 3 December 2018

Extended abstract deadline: 15 Feb. 2019

Initial announcement published: May 2018

${ }^{\star}$ Seventh AMS Symposium on the Joint Center for Satellite Data Assimilation (JCSDA), 6-11 January, Phoenix, Arizona

Session topic proposal deadline: 1 April 2018

Abstract deadline: 1 August 2018

Preregistration deadline: 3 December 2018

Extended abstract deadline: 15 Feb. 2019

Initial announcement published: May 2018

${ }^{*}$ Seventh Symposium on the MaddenJulian Oscillation and Sub-Seasonal Monsoon Variability, 6-11 January, Phoenix, Arizona

Session topic proposal deadline: 1 April 2018

Abstract deadline: 1 August 2018

Preregistration deadline: 3 December 2018

Extended abstract deadline: 15 Feb. 2019

Initial announcement published: May 2018

${ }^{*}$ Fifth Symposium on High Performance Computing for Weather, Water, and Climate, 6-11 January, Phoenix, Arizona

Session topic proposal deadline: 1 April 2018

Abstract deadline: 1 August 2018

Preregistration deadline: 3 December 2018

Extended abstract deadline: 15 Feb. 2019

Initial announcement published: June 2018

${ }^{*}$ Fourth Symposium on U.S.-International Partnerships, 6-11 January, Phoenix, Arizona

Session topic proposal deadline: 1 April 2018

Abstract deadline: 1 August 2018

Preregistration deadline: 3 December 2018

Extended abstract deadline: 15 Feb. 2019

Initial announcement published: May 2018
${ }^{*}$ Second Conference on Earth Observing SmallSats, 6-11 January, Phoenix, Arizona

Session topic proposal deadline: 1 April 2018

Abstract deadline: 1 August 2018

Preregistration deadline: 3 December 2018

Extended abstract deadline: 15 Feb. 2019

Initial announcement published: May 2018

${ }^{*}$ Tropical Cyclones and Extreme Monsoon Precipitation: Prediction, Impacts, and Communication, 6-11 January, Phoenix, Arizona

Session topic proposal deadline: 1 April 2018

Abstract deadline: 1 August 2018

Preregistration deadline: 3 December 2018

Extended abstract deadline: 15 Feb. 2019

Initial announcement published: May 2018

${ }^{*}$ Mesoscale Processes/Dynamics that Drive Extreme Events from Three Perspectives: Understanding, Prediction, Projection, 6-11 January, Phoenix, Arizona

Session topic proposal deadline: 1 April 2018

Abstract deadline: 1 August 2018

Preregistration deadline: 3 December 2018

Extended abstract deadline: 15 Feb. 2019

Initial announcement published: May 2018

${ }^{\star}$ Phased Array Radar Symposium, 6-11 January, Phoenix, Arizona

Session topic proposal deadline: 1 April 2018

Abstract deadline: 1 August 2018

Preregistration deadline: 3 December 2018

Extended abstract deadline: 15 Feb. 2019

Initial announcement published: June 2018

${ }^{*}$ Special Symposium on Meteorological Observation and Instrumentation, 6-11 January, Phoenix, Arizona

Session topic proposal deadline: 1 April 2018

Abstract deadline: 1 August 2018

Preregistration deadline: 3 December 2018

Extended abstract deadline: 15 Feb. 2019

Initial announcement published: May 2018
${ }^{*}$ Special Symposium on Catalyzing Innovation in Weather Science Internationally, 6-11 January, Phoenix, Arizona

Abstract deadline: 1 August 2018

Preregistration deadline: 3 December 2018

Extended abstract deadline: 15 Feb. 2019

Initial announcement published: June 2018

*Special Symposium on Global and Mesoscale Models: Updates and Center Overviews, 6-11 January, Phoenix, Arizona

Session topic proposal deadline: 1 April 2018

Abstract deadline: 1 August 2018

Preregistration deadline: 3 December 2018

Extended abstract deadline: 15 Feb. 2019

Initial announcement published: May 2018

15th Conference on Polar Meteorology and Oceanography, 19-23 May, Boulder, Colorado

Session topic proposal deadline: TBD

Abstract deadline: 18 January 2019

Preregistration deadline: 19 April 2019

Extended abstract deadline: 21 June 2019

Initial announcement published: Aug. 2018

18th Conference on Mesoscale Processes, 29 July-1 August, Savannah, Georgia

Abstract deadline: 25 March 2019

Preregistration deadline: 14 June 2019

Extended abstract deadline: 28 June 2019

Initial announcement published: Sep. 2018

47th AMS Conference on Broadcast Meteorology/Fifth Conference on Weather Warnings and Communication, 12-14 June, San Diego, CA Session topic proposal deadline: TBD Abstract deadline: 13 February 2019 Preregistration deadline: 8 May 2019 Extended abstract deadline: 12 July 2019

\footnotetext{
${ }^{\star}$ An exhibit program will be held at this meeting.
} 
39th International Conference on Radar Meteorology, 16-20 September, Nara Kasugano International Forum, Nara, Japan

Abstract deadline: 20 May 2019

Preregistration deadline: 1 August 2019

Extended abstract deadline 16 August 2019

Initial announcement published: Oct. 2018

23rd AMS Satellite Meteorology, Oceanography and Climatology Conference/2019 EUMETSAT Meteorological Satellite Conference/NOAA Satellite Conference, 28 September-4 October 2019, Boston, MA

Session topic proposal deadline: TBD

Abstract deadline: 8 April 2019

Preregistration deadline: 2 August 2019

Extended abstract deadline: 8 Nov. 2019

Initial announcement published: Aug. 2018
MEETINGS OF INTEREST

2018

\section{OCTOBER}

Sixth SPARC General Assembly, 1-5 October, Kyoto, Japan

NOAA 43rd Climate Diagnostics and Prediction Workshop, 23-25 October, Santa Barbara, California

\section{NOVEMBER}

Northeast Regional Operational Workshop (NROW) XIX, 7-8 November, Albany, New York

\section{9}

\section{MARCH}

A\&WMA Eighth Specialty Conference on Air Quality Models, 19-21 March, Durham, North Carolina
JUNE

AMOS Annual Meeting 2019 and the International Conference on Tropical Meteorology and Oceanography, 11-15 June, Darwin, Australia

\section{NOVEMBER}

Fourth COSPAR Symposium, 4-8 November, Herzliya, Italy

\section{0}

\section{AUGUST}

43rd COSPAR Scientific Assembly and Associated Events, 15-23 August, Sydney, Australia

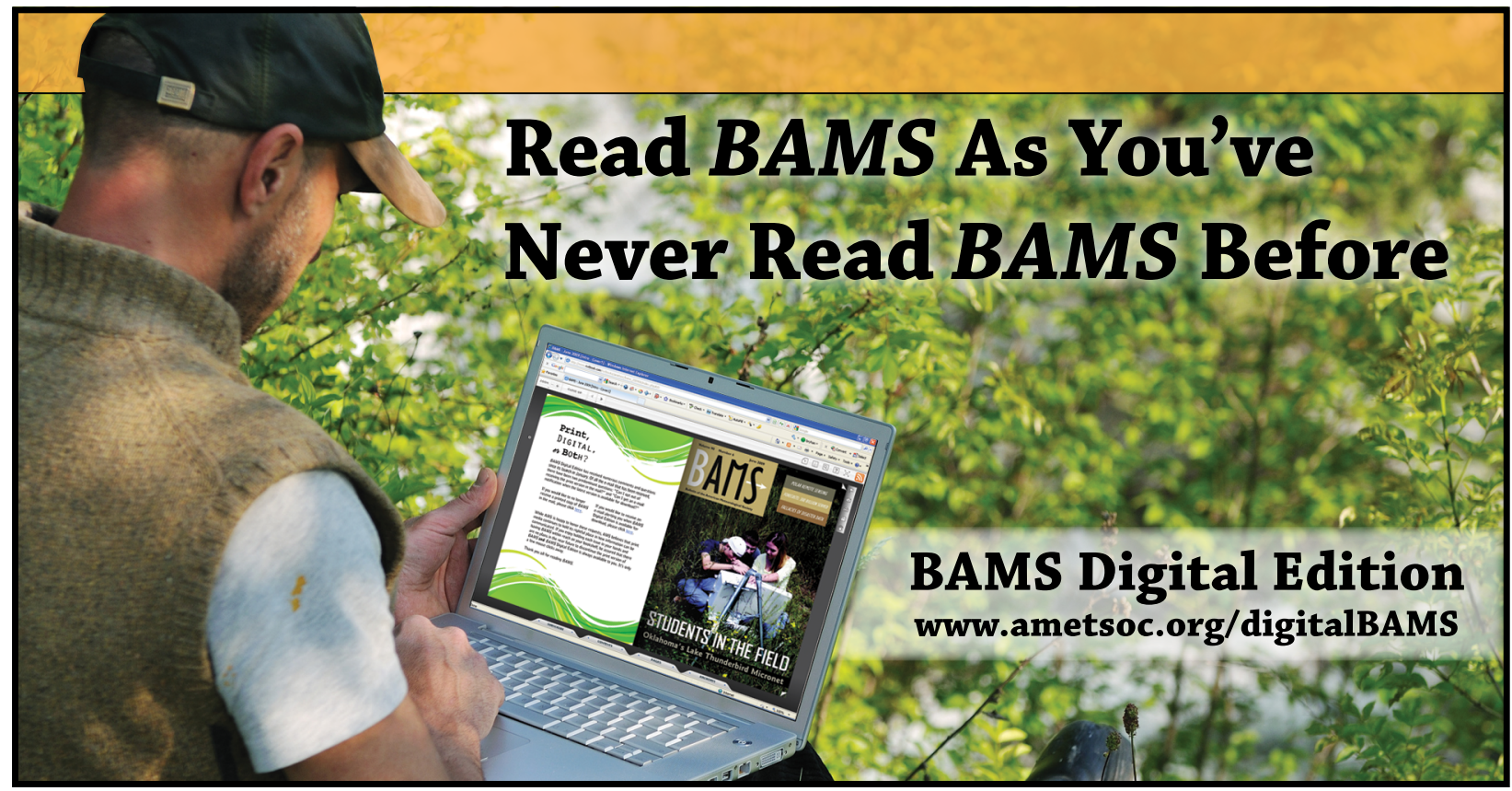




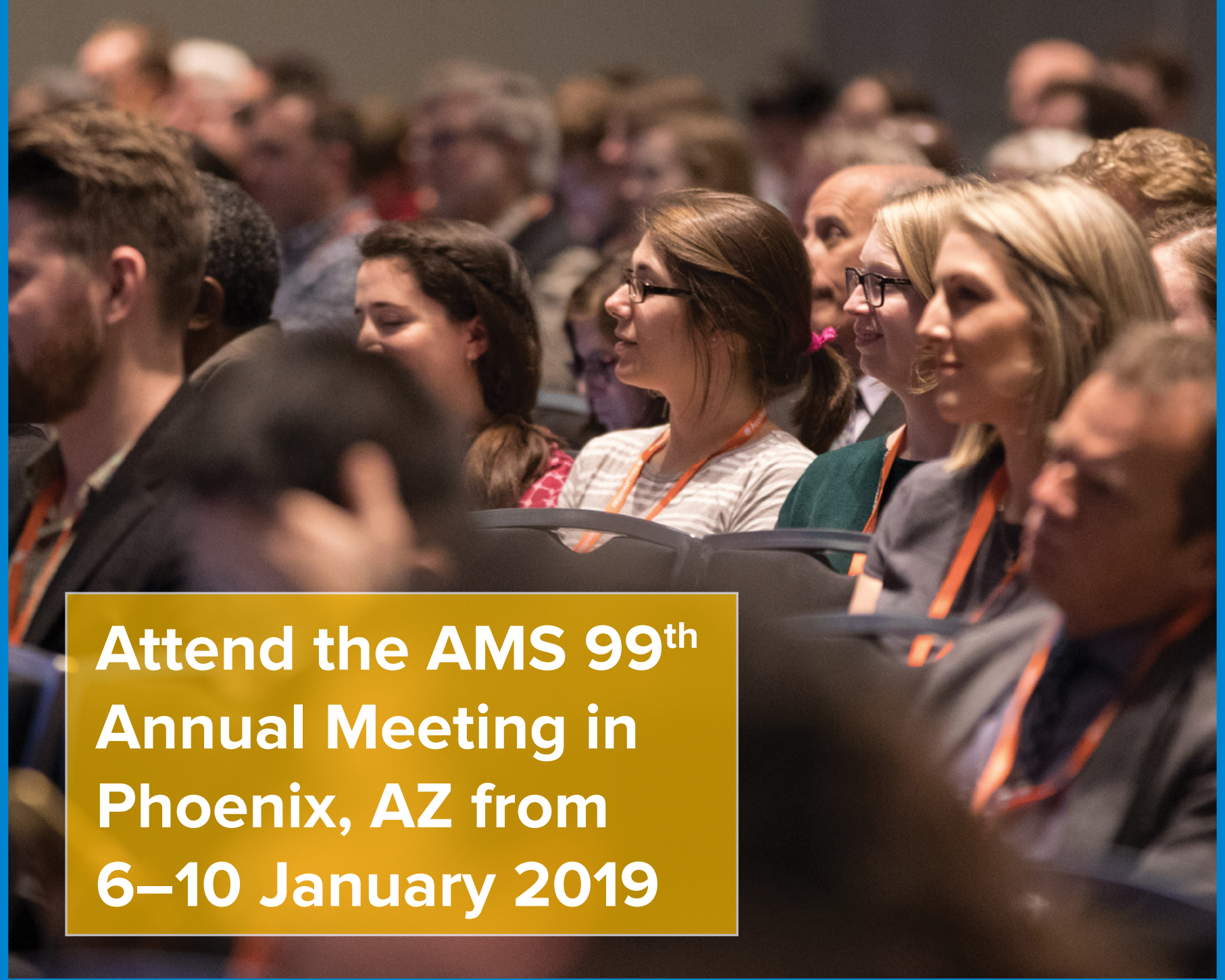

This year's theme is

Understanding and Building Resilience to Extreme Events by Being Interdisciplinary, International, and Inclusive (III)

Don't miss 30+ conferences and symposia, networking opportunities, short courses, and hundreds of exhibitors. Early registration deadline is 1 December.

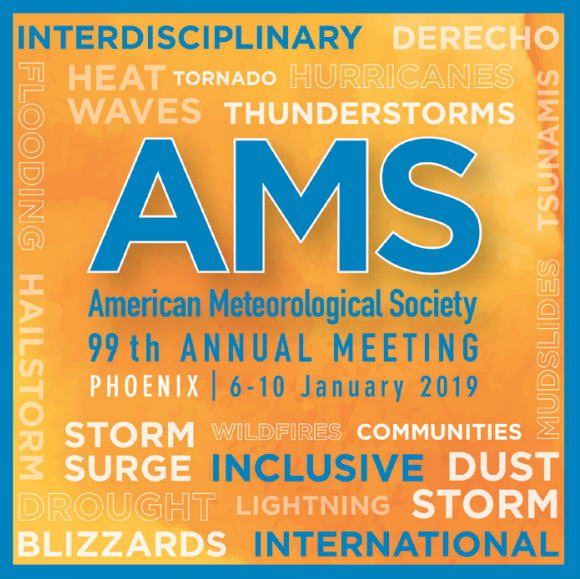

annual.ametsoc.org/2019 